face-to-face (F2F) recruitment programme in 2018 with our first remote recruitment programme in 2020. In a world of fast paced technology, we reflect on what lies in the horizon for international psychiatric recruitment.

Method. The recruitment programme was organised by 4 psychiatric trainees affiliated to each mental health trust. A two-day remote programme on the Zoom platform comprising of 45-minute slots was created. Through their own experiences of inspirational speakers, trainees contacted speakers representing different specialities, teaching styles and philosophical outlooks.

Pre and Post Programme questionnaires and certificates of attendance were shared with speakers and attendees. These were compared with Pre and Post Programme questionnaires from the F2F event in 2018.

Result. When compared to the 2018 F2F programme, the 2020 virtual recruitment programme attracted a higher number and wider variety of applicants, in gender (62\% female/38\%male), nationality (UK 79\%/Non-UK 22\%), as well as wider distribution in age, UK deanery and training position. Despite the elimination of cost, the quality of teaching was rated higher than F2F due to the availability of high quality speakers $(100 \%$ would recommend to a friend; $72 \%$ rated excellent). Whilst the programme was effective in changing minds, this did not exceed F2F recruitment rates. F2F recruitment feedback focussed on inclusion of subspecialities, whereas virtual programme feedback focussed on ways to enhance interaction. Feedback focussed on technological applications such as interactive quizzes, breakout rooms, play-acting, and having the benefit of clinical vignettes or speakers' personal stories to bring talks to life.

Conclusion. The use of remote technology transcended geographical and demographic frontiers. A variety of high-quality speakers, directly appealing to an international cohort were sourced, at no monetary cost. In future, the budget will be used in interactive applications, and time-limited session recordings. As the participants hungered for personal connections, we recommend a blended programme, with links to taster sessions, retaining the advantages of both strategies.

\section{School mental health best practices institute; capacity building of teachers in mental health literacy in Pakistan}

Maria Khan ${ }^{1 \star}$, Aisha Sanober Chachar ${ }^{2}$, Wamiq Ali $^{3}$ and Ayesha Mian ${ }^{1}$

${ }^{1}$ The Aga Khan University Hospital; ${ }^{2}$ Alleviate Addiction Suffering Trust and ${ }^{3}$ Dow University of Health Sciences

${ }^{*}$ Corresponding author.

doi: 10.1192/bjo.2021.407

Aims. Mental health disorders in children are largely unrecognized in low- and middle-income countries like Pakistan. Teachers, due to their interactions and time spent with children, are important elements in promoting child mental health. Despite this, little importance is given to school mental health $(\mathrm{SMH})$ in the country, and teachers' training in SMH is almost non-existent. With less than ten child and adolescent psychiatrists, recruiting teachers is vital to provide mental health care to children and adolescents, the majority of the country's population. This study aims to evaluate the effectiveness of a SMH training intervention for teachers in Pakistan.

Method. A 3-day training intervention was planned for school teachers in collaboration with International Association of Child and Adolescent Psychiatry and Allied Professions. The School Mental Health curriculum by the World Health Organization and Stan Kutcher's Mental Health Literature were adapted after literature review and discussions with experts, and administered as Blended Learning. The intervention was evaluated using preworkshop, post-workshop and overall feedback surveys. SPSS 25.0 (IBM Corp., Armonk, N.Y., USA) software was used for descriptive analysis. For open ended questions, central themes were identified, tabulated, and analyzed descriptively.

Result. A total of 63 participants registered for the workshop. The participants' mean age was 36.0 years, with $86 \%$ women and $14 \%$ men. Participants were mostly teachers, however, principals, administrators and counsellors also attended. Participants' reasons for attending were that they wanted to 'increase their knowledge' and learn 'practical management' of mental health issues. When asked about student wellbeing, $43 \%$ participants said it was a neglected area. Overall, $86.9 \%$ of participants felt the objectives were met well or very well and $87.61 \%$ stated there was adequate time for discussion. In addition, $90.47 \%$ participants responded that facilitators explained concepts well and $94.39 \%$ said facilitators answered questions well. Half of all Blended Learning activities were viewed by more than $50 \%$ of participants. Activity views decreased by $63.41 \%$ from the pre-workshop activities to day 3 activities. Improvements suggested by participants included taking a more problem-solving approach and focusing on the local context.

Conclusion. Evidence-based SMH interventions that train teachers are much-needed in the local resource-constrained settings. This intervention met its objectives effectively, however, Blended Learning was not well-received. We have studied learning analytics and identified the potential learner's profile of teachers as students. Adult learning principles should be implemented in future endeavours. This is a flagship project for future international collaborations between mental health professionals for cross-cultural knowledge exchange.

\section{Developing psychiatric resources for the new foundation programme curriculum}

Drew Kinmond ${ }^{1 \star}$, Fiona Hynes ${ }^{1}$ and Aqib

(Mohammad) Hussain ${ }^{2}$

${ }^{1}$ Reaside Clinic, Birmingham and Solihull Mental Health Foundation Trust and ${ }^{2}$ Birmingham Sollihull Mental health foundation trust ${ }^{*}$ Corresponding author.

doi: 10.1192/bjo.2021.408

Aims. Our aim was to develop an easily accessible, relevant and deliverable resource to meet the training requirements of the new foundation curriculum for Foundation Trainees in the West Midlands. The virtual resource needed to provide information at the correct knowledge depth, whilst also being flexible enough to allow trainees to access the materials despite the challenges of remote working. The West Midlands currently holds approximately 1,300 places for foundation training with an increase in numbers planned for 2023 and 2024.

Method. The United Kingdom Foundation Programme (FP) is a two-year structured, supervised training programme of learning in the workplace developed to prepare medical graduates for speciality training. The Foundation Curriculum is currently being updated in line with the GMC Standards for Post Graduate Curricula to reflect the developing and contemporaneous training needs of doctors and is expected to go live in August 2021.

Though the foundation curriculum is broad and does not usually include specific diseases, it is recognised that mental health disorders are common and are frequently missed. The new 